\title{
Dynamics of Electro-Optically Active Interfaces via Electron Holography and Femto-Second NSOM
}

Pradyumna Prabhumirashi, G. Shekhawat and Vinayak P. Dravid, Department of

Materials Science \& Engineering, NU Center for Nanotechnology, NUANCE User

Facilities, Northwestern University, Evanston, IL 60208, USA

Numerous electronic and electro-optical devices derive their scientifically appealing and technologically important properties and phenomena via nanoscale spatially varying electrostatic fields, and the complex interaction of polarization with light propagation.[1] In electrically active systems, interfaces and defects exhibit electrostatic potential barriers which provide effective potential barrier to charge transport or its break-down signals onset of nonlinear transport. In electro-optical devices, there is a remarkable coupling between electrodynamic polarization and light propagation, facilitated by external applied bias. As the need for enhanced speed with minimal losses for communication and data transfer increases, there are considerable challenges thus opportunities, to probe the dynamics of such relationship, now approaching 10's of GHz frequencies.

The role of electron holography to decipher nature of charge trapping and distribution atand across interfaces which forms the centerpiece in electrically active systems, has been amply elucidated.[2] In many such cases, the transport of charge across these interfaces is mediated through potential barriers which form at the core of these interfaces. These "charged" interfacial phenomena present ideal problems for electron holography. As a significant part of a broader program, we are investigating the interrelationship among interface variables (structure, chemistry and electronic structure) and interface-induced properties (varistor behavior, ferroelectric fatigue and switching) in model electroceramics such as $\mathrm{SrTiO}_{3}$ and PZT, BTO FE thin films.

Of particular interest is the adjustment and distribution of electrostatic potential under applied in-situ bias. This, however, poses stringent requirements for electron holography experiments. We have been able to develop lithography protocol for patterning transport electrodes (Figure 1) directly on TEM-ready thin foils, which enables both, transport property measurements as well as in-situ bias for holography - all inside the TEM.

Using such in-situ bias approach, we have been able to demonstrate activation of trapped GB charge at SrTiO3 GBs. Further, under applied bias, we have observed formation of (what we believe to be) oxygen vacancy defects at the electrode-Ferroelectric interfaces (see Figure 2). Such accumulation of additional charge contributes to the depolarization field, which is the likely cause of ferroelectric fatigue. Remarkably, accumulation of charge is apparently absent when the bottom electrode is made of conducting oxides, which are efficient scavengers of oxygen vacancies.

While electron holography is amenable to observing electronic phenomena at TV-rate, many useful electro-optic phenomena occur at $\mathrm{GHz}$ frequencies. To probe such ultra-fast phenomena we have developed a combination of NSOM with f-s laser system to enable observation of electro-optic phenomena at nano and pico-second range. The idea is to couple information gained by static or biased electron holography and correlate with that obtained via NSOM. The presentation will explore potential synergy between such disparate techniques and frequencies, and emphasize the complementary yet uniquely powerful role of electron holography in problems of interfaces and defects in electrooptically active phenomena and devices.[3] 


\section{REFERENCES:}

1. See, Grain Boundary Phenomena in Electroceramics, Adv. in Ceramics, Vol. 1, (Ed. L. Levinson and D. Hill, ACerS Publ., Columbus, OH, 1986). 2. K. D. Johnson and V. P. Dravid, Interface Science, 8 (2-3) 189-198 (2000). 3. This research is supported by US DOE (Grant No. DE-FG02-92ER45475).

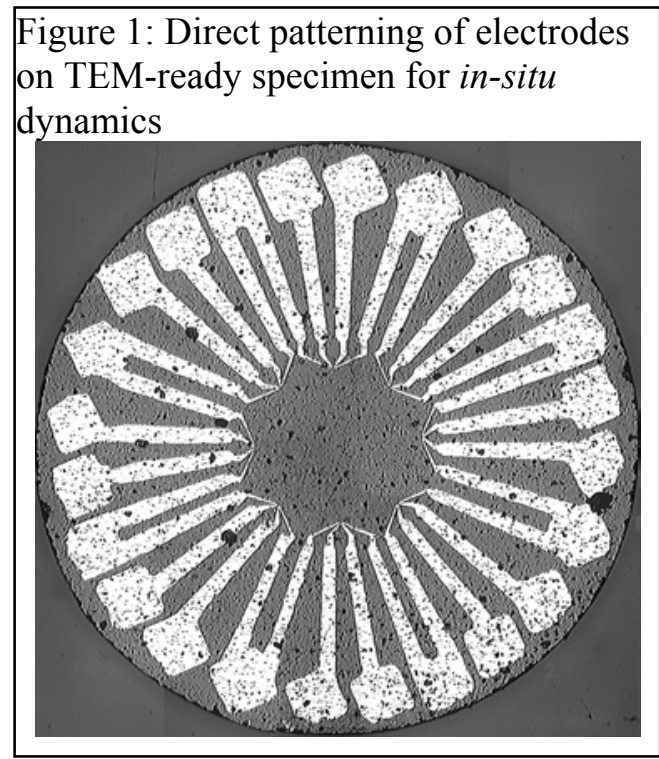

Fig.2B: Phase profile across PZT-Pt interface under in-situ bias- indicating possible presence of oxygen vacancies at interface

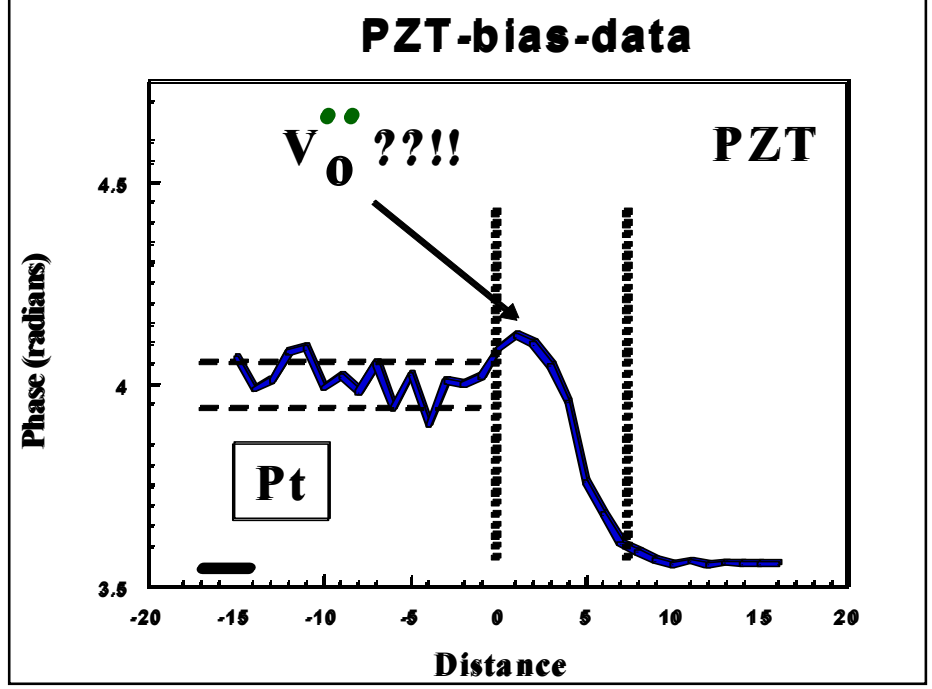

Fig. 2A: "Raw" holography phase image of PZT-Pt interface. Boxed area is used for potential profiles.

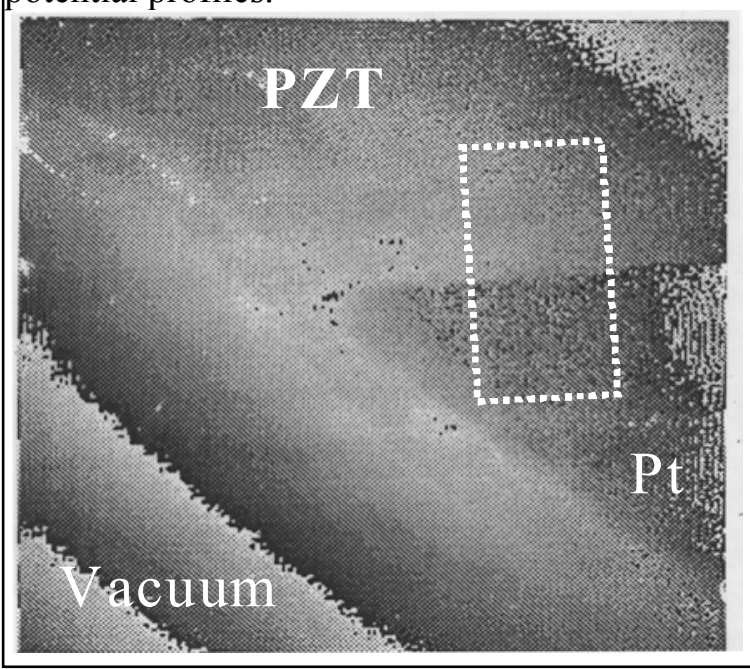

Fig.2C: Phase profile across PZT-SrRuO3 interface. Note: No unusual peak at interface

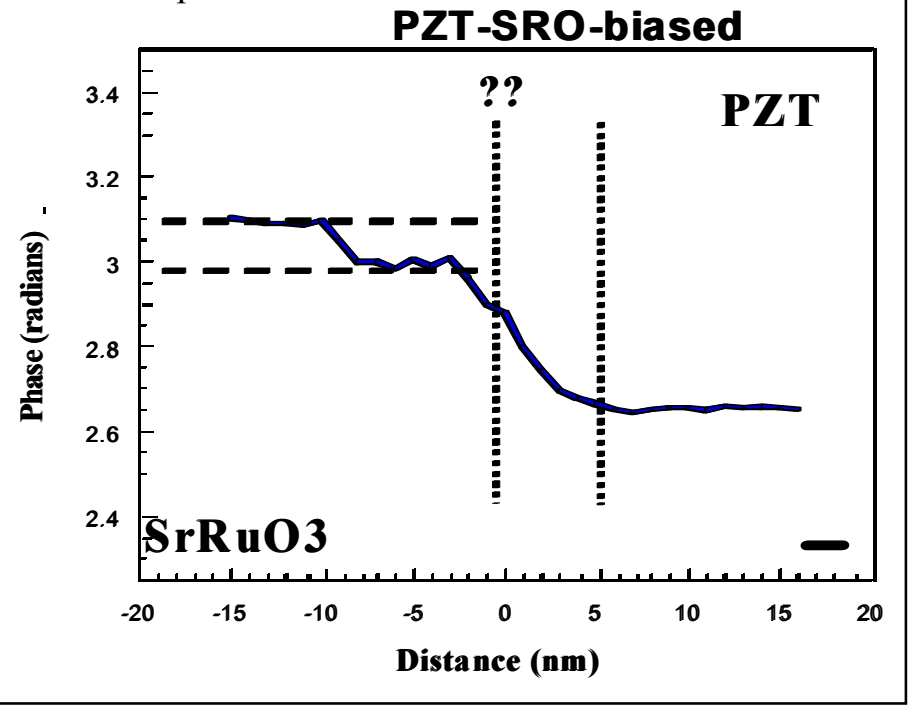

